\title{
Untersuchungen zur Herstellung von aromatischen Polyesterpolyolen für elastische Polyurethane
}

\author{
Stanislav Fulev, Rainer Langenstraßen, Gerhard Behrendt
}

\section{Einführung}

Aromatische Polyesterpolyole (APP) werden beispielsweise durch Umesterungen von Polyethylenterephthalat (PET) in Gegenwart von Diolen hergestellt. Daneben sind Verfahren bekannt, nach denen APP durch Umesterung von Dimethylterephthalat mit Diethylenglykol oder durch Veresterung von Phthalsäureanhydrid mit Polyethylenglykol oder mit Mischungen von Butan-1,4-diol mit weiteren Glykolen hergestellt werden.

APP können nach einem eigenen Verfahren diskontinuierlich [1] [2] oder kontinuierlich [3] hergestellt werden.

APP werden als Rohstoff zur Herstellung von Polyurethanen verwendet. Auf Grund ihrer Struktur und der daraus resultierenden Eigenschaften sind sie insbesondere zur Herstellung von flammgeschützten Polyurethan- sowie Polyisocyanurat-Hartschaumstoffen geeignet.

Grundsätzlich existieren vier Gruppen von aromatischen Polyesteralkoholen für den Bereich der PUR- und PIR-Hartschaumstoffe:

\begin{tabular}{|l|l|l|l|}
\hline Gruppe & $\begin{array}{l}\mathrm{OHZ} \\
(\mathrm{mg} \mathrm{KOH} / \mathrm{g})\end{array}$ & $\begin{array}{l}\text { Viskosität } 25^{\circ} \mathrm{C} \\
(\mathrm{mPa} \cdot \mathrm{s})\end{array}$ & Verwendung \\
\hline 1 & $400-420$ & 1000 & normale PUR-HS \\
\hline 2 & $360-380$ & 1200 & normale PUR-HS \\
\hline 3 & $300-320$ & $1800-2000$ & normale PUR-HS \\
\hline 4 & $220-240$ & $2200-3000$ & PIR-HS \\
\hline
\end{tabular}

In der vorliegenden Arbeit werden Untersuchungen dargestellt, die auf eine Erweiterung des Anwendungsbereiches in Richtung auf halbelastische PUR-Schaumstoffe zielen. Diese werden bei der Flammkaschierung zur Herstellung von Verbundwerkstoffen eingesetzt.

\section{Bisherige Arbeiten}

Über an der Technischen Fachhochschule Wildau durchgeführte Untersuchungen zur Herstellung aromatischer Polyesterpolyole aus PET wurde bereits mehrfach berichtet [4] [5]. Evtimova et al. [4] beschreiben ein modifiziertes Verfahren zur Herstellung von aromatischen Polyesterpolyolen aus PET unter Verwendung eines Oligoesterkondensats (OEK, Abfallprodukt aus der Polyester-Herstellung) und langkettiger Glykole sowie gegebenenfalls unter Zusatz von Dicarbonsäuren. Es werden Polyesterpolyole mit einem breiten Eigenschaftsspektrum erhalten, die zu PUR- und PIR-Hartschaumstoffen und Beschichtungsmaterialien verarbeitet werden.

Das eingesetzte Oligoesterkondensat besteht aus folgenden Komponenten:
40 - $60 \%$ Terephthalsäureester,

ca. $1 \%$ freie Terephthalsäure,

5 - $20 \%$ gebundenes Ethylenglykol,

1 - $33 \%$ freies Ethylenglykol,

1 - $5 \%$ gebundenes Diethylenglykol,

1 - $5 \%$ freies Diethylenglykol,

0,2 - $2 \%$ langkettige Glykole,

0,3 - $1 \%$ metallorganische Verbindungen (Katalysatoren).

Folgende Eigenschaftsbereiche der Polyesterpolyole wurden mit unterschiedlichen Reaktionsgemischen erhalten:

\begin{tabular}{|l|l|l|l|}
\hline Nr. & Ausgangsstoffe & OHZ $(\mathbf{m g ~ K O H} / g)$ & Viskosität (mPa·s) \\
\hline 1 & PET + OEK & $190-420$ & $13.000-165.000$ \\
\hline 2 & PET + OEK + Glykole & $170-410$ & $30.000-70.000$ \\
\hline 3 & $\begin{array}{l}\text { PET + OEK + Glykole + } \\
\text { Phthalsäure }\end{array}$ & $210-350$ & $7.800-60.000$ \\
\hline 4 & $\begin{array}{l}\text { PET + OEK + Glykole + } \\
\text { Adipinsäure }\end{array}$ & $130-200$ & $2.900-16.000$ \\
\hline
\end{tabular}

Die Polyole nach 1 . weisen eine hohe Viskosität und eine hohe Kristallisationsneigung auf, die für eine Verarbeitung zu Polyurethanen nachteilig sind. Eine Verbesserung wird durch Einsatz von Glykolen erreicht, wobei sich Diethylenglykol (DEG) als das geeignetste Glykol erwiesen hat. Durch Zusatz von Adipinsäure zu Reaktionsmischungen nach 2. wurde eine weitere, erhebliche Verminderung der Viskosität der Polyole erzielt [6]. Für die Polyole gilt generell, dass eine Viskosität von über $10.000 \mathrm{mPa} \cdot \mathrm{s}$ (in Abhängigkeit vom Verwendungszweck) für die wesentlichen Verarbeitungsverfahren von Nachteil ist.

Die PUR-Hartschaumstoffe, die aus APP nach 1. und 2. hergestellt wurden, weisen eine gute Dimensionsstabilität (bis $120^{\circ} \mathrm{C}$ ) und ein gutes Flammschutzverhalten auf, während Dimensionsstabilität und Flammschutz bei den aus APP nach 3. und 4. erhaltenen Schaumstoffen verringert werden.

Die nach den aufgeführten Verfahren herstellbaren Polyole sind außerdem geeignet zur Verarbeitung zu Beschichtungen und Vergussmassen sowie als Zusatzstoffe zu Polyester-PUR-Blockweichschaumstoff-Systemen. Für die alleinige Verwendung zur Herstellung elastischer Weichschaumstoffe sind sie auf Grund der dafür zu starren Molekülstruktur und der zu großen Kristallisationsneigung nicht geeignet.

In Grundlagenuntersuchungen zum Löseprozess und zur Umesterungsreaktion von PET in Glykol-OEK-Mischungen, die an der Technischen Fachhochschule Wildau ausgeführt werden [5], wurden optimale Lösegemische und -bedingungen erarbeitet, durch die die Lösezeiten in der Miniplantanlage von anfangs mehreren Stunden auf 10 bis 15 Minuten gesenkt werden. 
Während der Umesterung von PET in den untersuchten Glykolyse-Gemischen laufen zwei Vorgänge ab, die die wesentlichen Produktparameter Viskosität und Hydroxylzahl in entgegengesetzter Richtung beeinflussen, und zwar der Abbau der langen Kettenmoleküle, durch den die Viskosität der Lösung abnimmt, und die destillative Abtrennung der flüchtigen Nebenprodukte, insbesondere Ethylenglykol, andere niedermolekulare Verbindungen und Dioxan, die zu einer Zunahme der Viskosität und einer Abnahme der Hydroxylzahl des Produktes führt. Durch eine geeignete Wahl der Zusammensetzung des Glykolyse-Gemisches, der Reaktionstemperatur und der Reaktionsdauer bzw. der Destillatmenge als Abbruchkriterium können Produkteigenschaften erhalten werden, die die Zielvorgaben, insbesondere eine geeignete Paarung von Hydroxylzahl und Viskosität, erfüllen. Die Untersuchungen haben eine Palette von APP mit Parametern ergeben, mit denen sie für eine Vielzahl von Anwendungsfällen geeignet sind. Ebenso wie die oben genannten APP [4] sind die in [5] beschriebenen APP zur Herstellung von PUR- bzw. PIRHartschaumstoffen, Beschichtungen und Vergussmassen gut geeignet, während eine Verarbeitung zu elastischen Schaumstoffen nicht möglich ist.

Auf der Basis der an der TFH Wildau entwickelten Verfahren zur Herstellung von APP durch Glykolyse von PET ist in Mexiko eine 1-t-Anlage mit unserer Beteiligung installiert und in Betrieb genommen worden.

Über die gegenwärtig bearbeitete konstruktive und verfahrenstechnische Entwicklung eines kontinuierlich arbeitenden Reaktors zur Herstellung von APP an der TFH Wildau [5] wird in einer späteren Veröffentlichung berichtet.

\section{Aufgabenstellung}

Die Erzeugung langkettiger APP, die so modelliert sind, dass sie für die Herstellung halbelastischer Polyurethane benutzt werden können, ist bisher wenig untersucht worden. Folgende Parameter müssen von den Polyolen für diesen Verwendungszweck erfüllt werden:

- Hydroxylzahl < $100 \mathrm{mg} \mathrm{KOH/g}$

- Viskosität $\eta_{75}{ }^{\circ} \mathrm{C}<1500 \mathrm{mPa} \cdot \mathrm{s}$

- geringe Kristallisationsneigung (durch geringe Ausbildung von Strukturviskosität)

Die übliche Bezugstemperatur für Viskositätsmessungen von Polyolen ist $25^{\circ} \mathrm{C}$. Da jedoch Polyole mit OH-Zahlen unter $100 \mathrm{mg} \mathrm{KOH} / \mathrm{g}$ so hohe Viskositäten haben, dass ihr Einsatz zur Herstellung von Polyurethanen erst oberhalb ca. $50-60{ }^{\circ} \mathrm{C}$ möglich ist, werden deren Viskositäten häufig bei $75^{\circ} \mathrm{C}$ gemessen.

Das Ziel der Herstellung von APP mit den genannten Kennwerten soll durch Untersuchungen erreicht werden, die sich mit der Umesterungsreaktion von PET mit verschiedenen Glykolyse-Gemischen auf der Basis von Oligoesterkondensaten, verschiedenen langkettigen oder verzweigten Glykolen und Adipinsäure als aliphatischer Dicarbonsäure befassen.

Die auf den Lösevorgang folgenden Umsetzungen des PET sind Umesterungen mit Glykolen, wobei auf Grund des Kondensationsgleichgewichts ein niedriger siedendes, d. h. kleineres Glykol, abdestilliert und so aus dem Gleichgewicht entfernt wird. Weiterhin ist bei Umesterungen in der Regel eine Katalyse erforderlich, um diese Reaktion in vertretbarer Zeit zu einem bestimmten Zielprodukt bringen zu können [3] [7] [8] [9]. Als Katalysatoren dienen meist Metalloxide oder metallorganische Verbindungen, z. B. Titantetrabutylat, die der Reaktionsmischung direkt oder in Form des Oligoesterkondensats zugesetzt werden können. Das verwendete Glykol oder Glykolgemisch ist für die Eigenschaften des Endprodukts von entscheidender Bedeutung. Wird ein einheitliches Glykol verwendet, erhält man regelmäßige Kettenstrukturen, die zu hohen Viskositäten führen können. Werden Glykolgemische verwendet, werden bei geeigneter Wahl der Bausteine niedrigere Viskositäten und eine Reduzierung der Ausbildung von Überstrukturen erreicht.

Verwendet man langkettigere, verzweigte Glykole zur Glykolyse, so erhält man verglichen mit PET flexiblere Moleküle mit geringerer Kristallisationsneigung. Dadurch und durch die Verwendung von Glykol-Gemischen, die zu uneinheitlichen Molekülen führen, kann die Viskosität der erhaltenen Polyole bei gleicher mittlerer Molmasse verringert und ihre Kristallisationsneigung stark vermindert werden.

Die bisher beschriebenen Ergebnisse [4] [5] betreffen APP mit Eigenschaften, die sie für die Herstellung von PUR-Hartschaumstoffen und Polyisocyanurat-Schaumstoffen geeignet machen. Für jeden Verwendungszweck können Polyole hergestellt werden, deren Hydroxylzahlen im Idealbereich liegen. Dabei kann in Abhängigkeit vom technischen Prozess in jedem einzelnen Fall die erwünschte Viskosität bei stets niedrig gehaltener Säurezahl $(<1,0$ $\mathrm{mg} \mathrm{KOH} / \mathrm{g}$ ) erreicht werden.

Eine Flexibilität der APP-Molekülketten, wie sie zur Verwendung für elastische PUR-Schaumstoffe erforderlich ist, wird jedoch auf diese Weise noch nicht erreicht. Daher wird hier untersucht, welchen zusätzlichen Einfluss eine gegenüber bisherigen Arbeiten weitergehende Substitution der sehr starren Terephthalsäure-Einheiten durch aliphatische Dicarbonsäuren, z. B. Adipinsäure, hat, und wie die Eigenschaften der APP durch partielle Umesterung des PET mit langkettigen oder verzweigten Glykolen beeinflusst werden können.

Wie bekannt ist, besteht zwischen der Glasübergangstemperatur $\mathrm{T}_{\mathrm{g}}$ und den thermodynamischen Größen keine einfache Relation, wie sie beim Schmelzvorgang als Prozess erster Ordnung vorliegt. Mit Hilfe der Inkrementmethode von van Krevelen [10] oder nach der Methode von Kreibich über die Kohäsionsenergie $\mathrm{E}_{\text {coh }}$ [11] kann man jedoch $\mathrm{T}_{\mathrm{g}}$ für eine gegebene Struktur vorausberechnen. Durch die Normierung von $\mathrm{E}_{\mathrm{coh}}$ auf die Anzahl selbständig schwingungsfähiger Strukturelemente bei Polymeren ohne Seitengruppen erhält man einen linearen Zusammenhang zwischen $\mathrm{T}_{\mathrm{g}}$ und $\mathrm{E}_{\mathrm{coh}}$.<smiles>CC(C)OCCOCCOC(=O)c1ccc(C(=O)OCCOCCO)cc1</smiles>

Bild 1: Allgemeine Formel für ein aromatisches Polyesterpolyol 
Die Formel stellt eine vereinfachte allgemeine Struktur eines APP dar, das durch Glykolyse von PET mit Polyethylenglykolen unterschiedlicher Kettenlänge hergestellt wird. Für die hier behandelten APP gilt $1 \leq \mathrm{m} \leq 5$, bei Glykolyse von PET mit DEG gilt immer $\mathrm{k}=1$. In diesem Fall werden Produkte mit einer Hydroxylzahl von 420 bis $220 \mathrm{mg}$ $\mathrm{KOH} / \mathrm{g}$ und einem durchschnittlichen Molekulargewicht $\mathrm{M}_{\mathrm{n}}=265$ bis $510 \mathrm{~g} / \mathrm{mol}$ bei einer Funktionalität (Anzahl der $\mathrm{OH}$-Gruppen je Molekül) von $\mathrm{f}_{\mathrm{n}}=2$ erhalten.

Für den Zusammenhang zwischen der mittleren Molmasse $\mathrm{M}_{\mathrm{n}}$, der Hydroxyl-Konzentration OHZ und der Funktionalität $\mathrm{f}_{\mathrm{n}}$ gilt:

$$
\mathrm{M}_{\mathrm{n}}=\frac{\mathrm{f}_{\mathrm{n}} \cdot 56100}{\mathrm{OHZ}}
$$

\begin{tabular}{|l|l|}
\hline$M_{n}$ & mittlere Molmasse $(\mathrm{g} / \mathrm{mol})$ \\
\hline $\mathrm{OHZ}$ & Hydroxyl-Konzentration $(\mathrm{mg} \mathrm{KOH} / \mathrm{g})$ \\
\hline $\mathrm{f}_{\mathrm{n}}$ & Anzahl OH-Gruppen je Molekül (keine Dimension) \\
\hline 56100 & Molmasse von $\mathrm{KOH}(\mathrm{mg} \mathrm{KOH} / \mathrm{mol})$ \\
\hline
\end{tabular}

Das durch Glykolyse von PET mit DEG gewonnene Produkt hat eine Glasübergangstemperatur $\mathrm{T}_{\mathrm{g}} \approx 87^{\circ} \mathrm{C}$. Für $\mathrm{k}=2$ (Triethylenglykol) wird durch theoretische Berechnung $\mathrm{T}_{\mathrm{g}} \approx 75^{\circ} \mathrm{C}$ erhalten. Wenn ein Material mit einem k=10 (etwa durch Zusatz von PEG 400 zur Glykolysemischung) erzeugt wird, dann sollte sich theoretisch ein $\mathrm{T}_{\mathrm{g}}$ $\approx 0{ }^{\circ} \mathrm{C}$ ergeben, $\mathrm{d}$. h. das Material würde sich dann im elastischen Bereich befinden. Bild 2 zeigt die funktionelle Abhängigkeit der Glasübergangstemperatur von k für die angegebene Struktur.

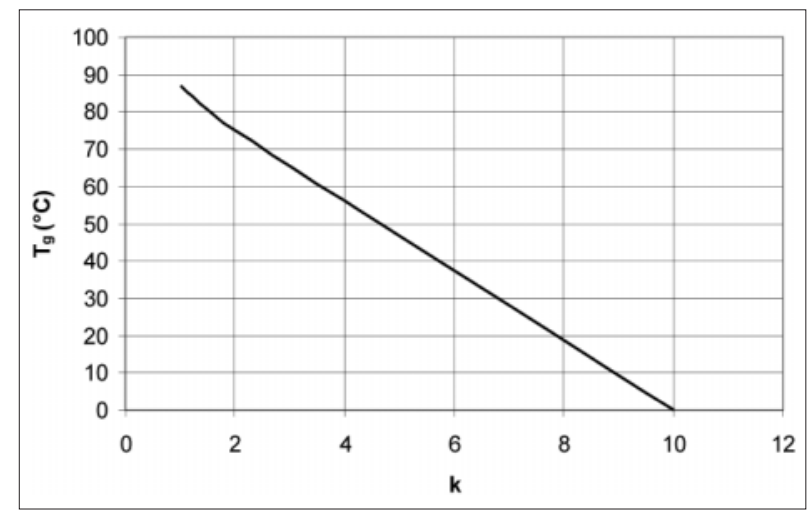

Bild 2: Abhängigkeit der Glasübergangstemperatur von der Kettenlänge des gebundenen Polyethylenglykols (s. Bild 1)

Über erste Untersuchungen in dieser Richtung mit dem Ziel, aromatische Polyesterpolyole herzustellen, die ein für die Verwendung als Ausgangsstoff für elastische Polyurethane erforderliches hohes Molekulargewicht und trotzdem eine niedrige Viskosität besitzen, wird hier berichtet.

\section{Allgemeine Versuchsvorschrift}

Die Komponenten des jeweiligen Glykolyse-Gemisches werden abgewogen und in die Vorratsbehälter eingefüllt. Das Oligoesterkondensat wird durch Erwärmen des Behälters auf $150{ }^{\circ} \mathrm{C}$ flüssig gehalten. Aus den Vorratsbehältern werden die Komponenten der Glykolysemischung
(OEK, Glykole) in den geschlossenen Miniplant-Reaktor gepumpt, wobei die Leitungen und die Pumpe für OEK beheizt sind. Die Reaktionsmischung wird unter Rühren auf die vorgesehene Lösetemperatur erwärmt. Der Gasraum des Reaktors wird während des gesamten Versuchs mit Stickstoff gespült. Das PET wird als Granulat mit einer Korngröße von ca. 3 bis $5 \mathrm{~mm}$ eingefüllt. Nach Lösen des PET wird gegebenenfalls Adipinsäure langsam portionsweise zugegeben und die Mischung auf die jeweilige Reaktionstemperatur erwärmt. Diese Temperatur wird gehalten, bis die vorgesehene Reaktionszeit oder Destillatmenge erreicht ist. Anschließend wird auf ca. $100{ }^{\circ} \mathrm{C}$ abgekühlt und das fertige Produkt über ein Filter in eine Vorlage gesaugt und von dort abgefüllt.

\section{Ergebnisse und Diskussion}

Polyole zum Zweck der Herstellung halbelastischer Polyurethane müssen eine niedrige Hydroxylzahl (< ca. $100 \mathrm{mg} \mathrm{KOH} / \mathrm{g}$ ) aufweisen. Das ist gleichbedeutend mit einer hohen mittleren Molmasse (> ca. $1500 \mathrm{~g} / \mathrm{mol}$ ). Die Erzeugung langkettiger APP (nach Möglichkeit mit einem langkettigen Aliphatenteil) ist immer mit einer hohen Viskosität verbunden, was durch die hohe Kristallisierbarkeit der PET-Polyole bedingt ist. Damit jedoch das Polyol für die vorgesehene Anwendungstechnologie geeignet ist, muss seine Viskosität unterhalb von $1500 \mathrm{mPa} \cdot \mathrm{s}$ bei $75^{\circ} \mathrm{C}$ liegen.

Unter Berücksichtigung der Angaben zur Kinetik des Lösevorgangs und der Umesterungsreaktion von PET und der Zielparameter wurden Systeme entwickelt, die die Erzeugung eines optimalen Produkts ermöglichen. $\mathrm{Zu}$ diesem Zweck wurden in mehreren Schritten der Untersuchungen langkettige Polyethylenglykole und verzweigte Glykole sowie als aliphatische Dicarbonsäure Adipinsäure (ADS) eingesetzt.

\begin{tabular}{|l|r|r|r|}
\hline Nr. & FD001 & FD002 & FD003 \\
\hline Anteil PET (\%) & 50,9 & 49,5 & 48,3 \\
\hline Anteil DEG (\%) & 29,5 & 28,8 & 28,0 \\
\hline Anteil ADS (\%) & 15,2 & 17,3 & 19,3 \\
\hline Anteil OEK (\%) & 4,4 & 4,4 & 4,4 \\
\hline$\eta_{25}{ }^{\circ} \mathrm{C}(\mathrm{mPa} \cdot \mathrm{s})$ & 135.000 & 174.000 & 270.000 \\
\hline $\mathrm{OHZ}(\mathrm{mg} \mathrm{KOH} / \mathrm{g})$ & 175 & 150 & 130 \\
\hline $\mathrm{SZ}(\mathrm{mg} \mathrm{KOH} / \mathrm{g})$ & 1,7 & 2,7 & 4,6 \\
\hline
\end{tabular}

Tabelle 1: Ergebnisse zur Umesterungsreaktion in DEG-ADSMischungen (Reaktion bei $250^{\circ} \mathrm{C}, 4 \mathrm{~h}$ ). Versuche mit steigenden Mengen ADS

Die steigende Menge von ADS führt zu einer niedrigeren $\mathrm{OH}-Z a h l$. Das ist auf die Verringerung der OH-Konzentration durch Veresterung mit ADS zurückzuführen. Bei den Versuchen FD002 und FD003 sind im Reaktionssystem zu wenig OH-Gruppen vorhanden, deshalb bekommt man ein Produkt mit endständigen Säuregruppen und daher höherer Säurezahl. Bei diesen Produkten ist die Viskosität noch zu hoch, so dass im nächsten Schritt untersucht wird, welchen Einfluss die Verwendung eines verzweigten oder eines langkettigeren Glykols auf die Produkteigenschaften hat. 


\begin{tabular}{|l|r|r|r|r|r|r|}
\hline Nr. & FD74 & FD75 & FD76 & FD71 & FD82 & FD83 \\
\hline Anteil PET (\%) & 49,1 & 46,8 & 44,7 & 46,6 & 45,5 & 44,5 \\
\hline Anteil DEG (\%) & 33,4 & 31,8 & 30,5 & 22,4 & 21,9 & 21,4 \\
\hline Anteil ADS (\%) & 4,9 & 9,4 & 13,4 & 14 & 13,7 & 13,3 \\
\hline Zweitglykol & DPG & DPG & DPG & $\begin{array}{r}\text { PEG } \\
400\end{array}$ & $\begin{array}{r}\text { PEG } \\
400\end{array}$ & $\begin{array}{r}\text { PEG } \\
400\end{array}$ \\
\hline $\begin{array}{l}\text { Anteil Zweit- } \\
\text { glykol (\%) }\end{array}$ & 10,3 & 9,8 & 9,4 & 15 & 16,8 & 18,7 \\
\hline Anteil OEK (\%) & 2,3 & 2,1 & 2,0 & 2,0 & 2,1 & 2,1 \\
\hline$\eta_{25}{ }^{\circ} \mathrm{C}(\mathrm{mPa} \cdot \mathrm{s})$ & 4.800 & & n.m. & n.m. & & n.m. \\
\hline$\eta_{75}{ }^{\circ} \mathrm{C}(\mathrm{mPa} \cdot \mathrm{s})$ & & & & & 710 & \\
\hline OHZ (mg KOH/g) & 390 & 320 & 200 & 170 & 140 & 180 \\
\hline $\mathrm{SZ} \mathrm{(mg} \mathrm{KOH/g)}$ & 0,8 & 1,55 & 2,9 & 4,8 & 2,4 & 3 \\
\hline
\end{tabular}

(n.m.: nicht messbar, > 300.000 mPa.s)

Tabelle 2: Ergebnisse der Umesterungsreaktion mit DEG-ZweitglykolADS-Mischungen (Reaktion bei $250^{\circ} \mathrm{C}, 4 \mathrm{~h}$ ). Versuche mit steigenden Mengen ADS und anschließender Zugabe von DPG oder PEG 400

Die Versuchsreihe mit DPG wurde ebenfalls mit steigenden Mengen ADS durchgeführt. DPG hat im Vergleich zu DEG eine größere Molmasse und seitenständige Methylgruppen, die eine Kristallisation behindern können. Die Versuche haben jedoch ergeben, dass höhere Konzentrationen von ADS zu einer Verschlechterung des Verhältnisses OHZ/ Viskosität führen. Das beste Ergebnisse dieser Versuchsreihe wurde mit PEG 400 erhalten (Versuch FD82).

Deshalb wurde dieses System als Grundlage für weitere Modifizierungen ausgewählt.

\begin{tabular}{|l|r|r|r|}
\hline Nr. & FD84 & FD85 & FD86 \\
\hline Anteil PET (\%) & 51,6 & 50,3 & 49,1 \\
\hline Anteil DEG (\%) & 16,5 & 18,6 & 20,6 \\
\hline Anteil ADS (\%) & 10,3 & 10 & 9,8 \\
\hline Zweitglykol & PEG 400 & PEG 400 & PEG 400 \\
\hline Anteil Zweitglykol (\%) & 19,0 & 18,6 & 18,2 \\
\hline Anteil OEK (\%) & 2,6 & 2,5 & 2,3 \\
\hline$\eta_{25}{ }^{\circ} \mathrm{C}(\mathrm{mPa} \cdot \mathrm{s})$ & 200.000 & 158.000 & 123.000 \\
\hline OHZ (mg KOH/g) & 130 & 150 & 152 \\
\hline SZ (mg KOH/g) & 4,8 & 3,1 & 6,3 \\
\hline
\end{tabular}

Tabelle 3: Ergebnisse zur Umesterungsreaktion in DEG-ZweitglykolADS-Mischungen (Reaktion bei $250^{\circ} \mathrm{C}, 4 \mathrm{~h}$ ). Versuche mit steigenden Mengen DEG

Die vorliegenden Daten machen deutlich, dass zur Vermeidung des Kristallisationsprozesses als eine der Ursachen für die hohe Viskosität ein neuer Weg gesucht werden muss. Die Reduzierung der Kristallisationsneigung gelingt mit der Anwendung von Neopentylglykol (2,2-Dimethyl-1,3-propandiol), das die Bildung von regelmäßigen Strukturen (Kristallisation) durch zwei seitenständige Methylgruppen verhindert.

\begin{tabular}{|l|r|r|r|}
\hline Nr. & FD87K & FD88K & FD89K \\
\hline Anteil PET (\%) & 47,0 & 44,9 & 43,0 \\
\hline Anteil DEG (\%) & 27,3 & 26,0 & 24,9 \\
\hline Anteil ADS (\%) & 18,8 & 18,0 & 17,2 \\
\hline Anteil NPG (\%) & 4,7 & 9,0 & 12,9 \\
\hline Anteil OEK (\%) & 2,2 & 2,1 & 2,0 \\
\hline$\eta_{25}{ }^{\circ} \mathrm{C}(\mathrm{mPa} \cdot \mathrm{s})$ & 114.200 & 31.500 & 34.800 \\
\hline$\eta_{75}{ }^{\circ} \mathrm{C}(\mathrm{mPa} \cdot \mathrm{s})$ & 2.200 & 1.400 & 6.800 \\
\hline $\mathrm{OHZ} \mathrm{(mg} \mathrm{KOH} / \mathrm{g})$ & 124 & 190 & 180 \\
\hline $\mathrm{SZ} \mathrm{(mg} \mathrm{KOH} / \mathrm{g})$ & 0,3 & 4,4 & 0,8 \\
\hline
\end{tabular}

Tabelle 4: Ergebnisse zur Umesterungsreaktion in DEG-ADS-Mischungen mit Zusatz von Neopentylglykol (Reaktion bei $250{ }^{\circ} \mathrm{C}, 4 \mathrm{~h}$ ).
Um die Wirkung der im OEK enthaltenen Katalysatoren mit industriell hergestellten Katalysatoren zu vergleichen und dabei das reduzierte Kristallisationsvermögen des APP zu erhalten, wurden analoge Versuche mit einem typischen metallorganischen Veresterungskatalysator, Titantetrabutylat $\left[\mathrm{Ti}(\mathrm{OBu})_{4}\right]$, anstelle von OEK durchgeführt.

\begin{tabular}{|l|r|r|r|r|}
\hline Nr. & FD93K & FD90K & FD91K & FD92K \\
\hline Anteil PET (\%) & 48,0 & 45,8 & 43,8 & 41,9 \\
\hline Anteil DEG (\%) & 27,9 & 26,6 & 25,4 & 24,4 \\
\hline Anteil ADS (\%) & 19,2 & 18,3 & 17,5 & 16,8 \\
\hline Anteil NPG (\%) & 4,8 & 9,2 & 13,2 & 16,8 \\
\hline Anteil Ti(OBu) $)_{4}(\%)$ & 0,1 & 0,1 & 0,1 & 0,1 \\
\hline$\eta_{25}{ }^{\circ} \mathrm{C}(\mathrm{mPa} \cdot \mathrm{s})$ & 95.000 & 50.800 & 96.000 & 101.600 \\
\hline$\eta_{75}{ }^{\circ} \mathrm{C}(\mathrm{mPa} \cdot \mathrm{s})$ & 20.000 & 1.670 & 6.000 & 11.600 \\
\hline $\mathrm{OHZ}(\mathrm{mg} \mathrm{KOH} / \mathrm{g})$ & 100 & 160 & 55 & 75 \\
\hline $\mathrm{SZ}(\mathrm{mg} \mathrm{KOH} / \mathrm{g})$ & 0,7 & 0,6 & 0,3 & 0,4 \\
\hline
\end{tabular}

Tabelle 5: Ergebnisse zur Umesterungsreaktion mit Ti(OBu)

Die so erhaltenen Daten bestätigen die Funktion des Oligoesterkondensats als Katalysator und damit die Wirtschaftlichkeit des Verfahrens.

\section{Zusammenfassung}

In der vorliegenden Arbeit ist eine Methode erarbeitet und erfolgreich erprobt worden, durch die aromatische Polyesterpolyole mit einer geeigneten Kombination aus hohem Molekulargewicht und einer für die Verarbeitung geeigneten, niedrigen Viskosität erhalten werden können. Die erhaltenen Parameter liegen dem geforderten Zielbereich nahe. Die Untersuchungen werden weitergeführt und gemeinsam mit Untersuchungen zur Herstellung von Polyurethanen aus diesen APP in einer späteren Veröffentlichung dargestellt.

\section{Abkürzungen und Symbole}

ADS Adipinsäure

APP aromatisches Polyesterpolyol

DEG Diethylenglykol

DPG Dipropylenglykol

NPG Neopentylglykol (2,2-Dimethyl-1,3-propandiol)

OEK Oligoesterkondensat

OHZ OH-Zahl, Hydroxylzahl (Konzentration an Hydroxylgruppen, Dimension mg KOH/g)

PEG 400 Polyethylenglykol mit einer mittleren Molmasse $400 \mathrm{~g} / \mathrm{mol}$

PET Polyethylenterephthalat

PIR Polyisocyanurat

PUR Polyurethan

SZ Säurezahl (Konzentration an Säuregruppen, Dimension mg KOH/g)

$\eta_{25}{ }^{\circ} \mathrm{C} \quad$ dynamische Viskosität (im Index Angabe der Messtemperatur) 


\section{Literatur}

[1] G. Behrendt, A. Lobeda, M. Pohl, Verfahren zur Herstellung von Polyesteralkoholen sowie Polyesteralkohole, WO 99/ 54380, DE-OS 19918650 (16.04.1998)

[2] G. Behrendt, M. Pohl, Polyester-Polymerpolyole für Polyurethane, DE-OS 19915125 (25.03.1999)

[3] G. Behrendt, R. Langenstraßen, B. Gebert, H.-D. Hunger, A. Diener, Th. Isenschmid, Verfahren und Vorrichtung zur Herstellung von Recyclat-Polyolen aus Polyestern, DE-OS 10 2004014163 (17.03.2003)

[4] R. Evtimova, Y. Loseva, K.-H. Schmidt, M. Wotzka, P. Wagner, G. Behrendt, Wissenschaftliche Beiträge der Technischen Fachhochschule Wildau, 2003, S. 19-25

[5] R. Langenstraßen, S. Fulev, A. Apel, B. Gebert, D. Lehmann, G. Behrendt, Wissenschaftliche Beiträge der Technischen Fachhochschule Wildau, 2004, S. 34-45

[6] G. Behrendt, M. Pohl, Polyesterpolyole mit niedriger Viskosität, DE-Anm. 19915128 v. 25. 3. 1999, innere Priorität DE-OS 19918650 (16.04.1998)

[7] U. R. Vaidya, V. M. Nadkarni, Polyester polyols from PET waste: effect of glycol type on kinetics of polyesterification, J. Appl. Polym. Sci. 38 (1991), S. 1179-1190

[8] F. P. La Mantia, M. Vinci, Recycling poly(ethylene terephthalate), Polymer Degradation and Stability 45 (1994), S. $121-125$

[9] U. R. Vaidya, V. M. Nadkarni, Ind. Eng. Chem. Res. 26 (1987), S. $194-198$

[10] D. W. van Krevelen, Properties of Polymers, Elsevier Sci. Publ. Co., Amsterdam 1976

[11] U. Kreibich, H. Batzer, Einfluss der Segmentstruktur und der Vernetzung auf den Glasübergang $\mathrm{T}_{\mathrm{g}}$. Angewandte Makromolekulare Chemie 83 (1979), S. 57-112

\section{Danksagung}

Die Verfasser danken der Firma Krauss-Maffai Kunststofftechnik GmbH, München, für die kostenlose Bereitstellung von PET-Ware und der Firma Trevira GmbH, Guben, für die kostenlose Bereitstellung von Oligoesterkondensat.

\section{Autoren}

Dipl.-Ing. Stanislav Fulev

Technische Fachhochschule Wildau

Fachbereich Ingenieurwesen/Wirtschaftingenieurwesen

Tel. +49 3375 508-406

stffulev@igw.tfh-wildau.de

Dr. Rainer Langenstraßen

Technische Fachhochschule Wildau

Fachbereich Ingenieurwesen/Wirtschaftingenieurwesen

Tel. +49 3375 508-502

rlangens@igw.tfh-wildau.de

Prof. Dr. Gerhard Behrendt

Technische Fachhochschule Wildau

Fachbereich Ingenieurwesen/Wirtschaftingenieurwesen

Tel. +493375 508-591

behrendt@vt.tfh-wildau.de 